\title{
Analysis and Simulations of the Effects of Non-linearity on the Radio Frequency Power Amplifier Modelling
}

\author{
Rajbir Kaur \\ Assistant Professor \\ ECE Deptt., Punjabi University \\ Patiala, Punjab, India
}

\author{
Manjeet Singh Patterh, Ph.D. \\ Professor \\ ECE Deptt., Punjabi University \\ Patiala, Punjab, India
}

\begin{abstract}
Radio frequency power amplifiers play a key role in transceivers for mobile communications and their linearity is a crucial aspect. In order to meet the linearity requirements dictated by the standard at a reasonable efficiency, the usage of a linearization technique is desired. For effective implementation of any linearization technique, accurate modelling of power amplifier is required. Due to its less complexity, memory polynomial has been widely used for modelling non-linear system with memory. So in this paper, memory polynomial has been used to model the wideband power amplifier. The effects of nonlinearity order and memory depth on the power amplifier modeling have also been simulated.
\end{abstract}

\section{Keywords}

Power amplifier; non-linearity; memory polynomial; predistortion; intermodulation products; bit error rate.

\section{INTRODUCTION}

In new generation mobile communication systems (LTE, WiMAX, WCDMA, CDMA2000 etc.), where spectrum efficient linear modulation formats are used, power amplifier (PA) linearity is a key requirement. As PA is one of the major sources of nonlinearity in communication systems, and its nonlinearity can significantly affect system performance. Since the linearity of a transmitter has to meet stringent spectral emission requirements, one has to accurately predict and compensate for the nonlinearities of the PA [1-2]. The transfer characteristic of PA is not linear up to the saturation point. The amplification decreases as the input power increases. There are many ways to express the nonlinear relationship mathematically. Here polynomials have been considered, such that a power series describes the relationship as,

$$
V_{\text {out }}(t)=a_{1} V_{\text {in }}(t)+a_{2} V_{\text {in }}^{2}(t)+a_{3} V_{\text {in }}^{3}(t)+-----
$$

The transfer characteristic now includes not only the linear term but also the higher order terms. In the equation 1, a third order polynomial represents the nonlinear transfer function. The second-order coefficient is positive and the third-order coefficient negative, which result in a compressive characteristic of the curve. The more the input signal grows, the larger the influence of the higher-order powers. Feeding an amplifier with a signal of some frequency, the output signal will include unwanted frequency components. This is referred to as AM/AM distortion [3], since the output amplitude will be distorted in relation to the input amplitude. The amplitude of the input signal affects the output signal phase. Increasing amplitude levels will introduce an increasing phase distortion on the output signal. The conversion of input power to output phase is called AM/PM distortion. Peaks will be clipped even with ideal amplifier if input exceeds maximum input power. With enough clipping, it appears as Gaussian noise at the receiver. The effects of clipping gives in-band distortion, degradation of bit error rate (BER) and higher error vector magnitude (EVM). Out of band radiations give adjacent channel interference (ACI) problems, like adjacent channel leakage ratio (ACLR) degradation, adjacent channel power ratio (ACPR) spreading etc. One of the most important measurements on RF signals for digital communication systems is the leakage power in the adjacent channels. Leakage power influences the system capacity as it interferes with the transmission in adjacent channels. Therefore it must be rigorously controlled to guarantee communication for all subscribers in a network. Thus the problem of peak to average power ratio (PAPR) is the major concern. Always there is tradeoff for the design and optimization of the PAPR reduction algorithm within the context of the EVM and ACLR.

EVM is in-band distortion causing high BER during reception of the transmitted data [4]. EVM gives a measure of the phase and amplitude distortion of a signal. It is one way of quantifying transmitter system performance and it is a measure of the difference between the measured and a reference signal. As shown in Figure 1 EVM is the magnitude of the error vector.

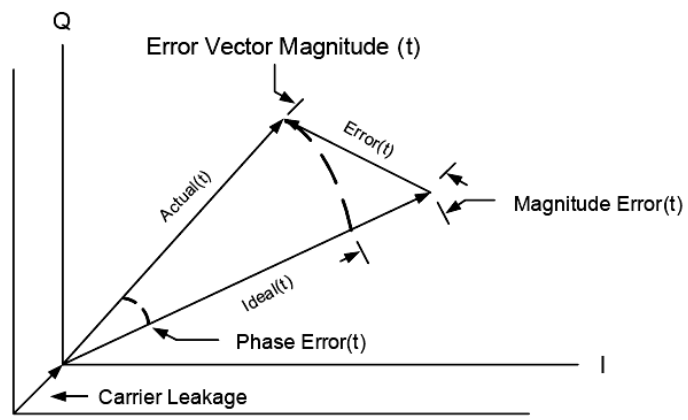

Fig 1: Error Vector Magnitude

EVM is the distance between the desired and actual signal vectors. From figure 1, the actual value of the constellation point can deviate from the ideal one significantly depending on PA nonlinearity. Mathematically EVM can be defined for each symbol of $\mathrm{K}$ as:

$$
\frac{|E(k)|}{\sqrt{\frac{1}{N}} \sum_{k=1}^{n}|s(k)|^{2}}
$$

Where $\mathrm{E}(\mathrm{k})$ is the error vector for symbol $\mathrm{k}, \mathrm{S}(\mathrm{k})$ is the ideal signal vector of the symbol $\mathrm{k}$ and $\mathrm{N}$ is the number of symbols. 
Therefore some linearization techniques are required to minimize the distortion on the transmit signal. Among all the linearization techniques, digital predistortion (DPD) is the most efficient and cost effective technique [5]. Memory polynomial PA model is a simplified model based on indirect learning architecture. This model is able to capture some of the memory effects, while reducing the number of parameters describing the model. Proposed memory polynomial model can be used for any wideband PA data. In this paper for modelling PA, the degree and order of memory polynomial can be varied to obtain the optimum result. The paper is organized as follows: section I provides the introduction to PA and non-linearity problem in PA, section II gives nonlinearity analysis of PA using Single-tone stimulus and Two-Tone stimulus analysis, section III gives memory polynomial model using indirect learning architecture, section IV gives the results for memory polynomial model and section $\mathrm{V}$ concludes the paper.

\section{NON-LINEARITY IN POWER AMPLIFIER}

Memory Polynomial model for power amplifier shows the non-linearity with memory effects [6]. This model can be designed with unity time delay taps and also with nonuniform time delay taps. Modelling is done by using data in discrete time domain. Signal is first converted from continuous to discrete by a method of sampling. If $V_{i n}(t)$ is the input signal to the sampler, then the output of sampler is:

$V_{\text {out }}(i)=V_{\text {in }}(i . T)$, Where $\mathrm{T}$ is the sampling period and sampling frequency is $F_{\text {samples }}=\frac{1}{T}$.

Let the baseband signal is

$$
Z_{\text {in }}(t)=V(t) \cdot \operatorname{Cos}\left[\left(\omega_{c} t+\theta(t)\right]\right.
$$

Here $\omega_{c}$ is the angular carrier frequency, $\mathrm{V}(\mathrm{t})$ is the amplitude and $\theta(t)$ is the phase of the signal. The envelop band width of this signal is much lower than the carrier frequency. This signal can be written in terms of in-phase and quadrature components as:

$$
Z_{\text {in }}(t)=I(t) \cdot \operatorname{Cos}\left(\omega_{c} t\right)-Q(t) \cdot \sin \left(\omega_{c} t\right)
$$

Where $I(t)=V(t) \cdot \operatorname{Cos}[\theta(t)]$

$$
Q(t)=V(t) \cdot \operatorname{Sin}[\theta(t)]
$$

Equation (4) can be re-written in complex form as:

$$
\begin{aligned}
& Z_{\text {in }}(t)=V(t) \cdot \operatorname{Cos}[\theta(t)] \cdot \operatorname{Cos}\left(\omega_{c} t\right)-V(t) \cdot \operatorname{Sin}[\theta(t)] \cdot \sin \left(\omega_{c} t\right) \\
& Z_{\text {in }}(t)=\operatorname{Re}\left[A(t) \cdot e^{j \omega_{c} t}\right]
\end{aligned}
$$

Here $A(t)$ is a base band signal and

$$
A(t)=V(t) \cdot e^{j \theta(t)}
$$

\subsection{Nonlinearity Analysis of Power Amplifier using Single-Tone Stimulus}

For a linear amplifier the output can be described as:

$$
Z_{o}(t)=G_{\text {Lin }} \cdot Z_{\text {in }}(t)
$$

where $G_{L i n}$ is a time independent linear amplifier gain. Practically due to non-linearity, the output of amplifier saturates at some value as the input signal amplitude is increased. Due to non-linearity, amplifier has a non-constant gain and non-linear phase. These amplifiers are called quasimemory-less amplifiers and described by the polynomial as

$$
Z_{o}(t)=\sum_{k=0}^{K} a_{k} Z_{i n}(t)^{k}
$$

$$
Z_{o}(t)=a_{0}+a_{1} \cdot Z_{i n}(t)+a_{2} \cdot Z_{i n}(t)^{2}+-------a_{k} \cdot Z_{i n}(t)^{k}
$$

In equation (11), $\mathrm{k}$ is the maximum polynomial order which shows the non-linearity of the amplifier. $a_{0}, a_{1}, a_{2}-----a_{k}$ are complex polynomial coefficients, which determine the exact shape of the input-out-put characteristics. For memory-less case, these polynomials are real values. By using trigonometric formulas, the quasimemory-less amplifier will produce new frequency components which are located at the harmonics ( $2 \omega, 3 \omega,-----K \omega)$ of the input signal.

From equation (7)

$$
Z_{\text {in }}(t)=V(t) \cdot \operatorname{Cos}\left[\omega_{c} t+\theta(t)\right]
$$

By substituting this value in equation 10,

$$
Z_{o}(t)=\sum_{k=0}^{K} a_{k} \cdot\left[V(t) \cdot \operatorname{Cos}\left(\omega_{c} t+\theta(t)\right)\right]^{k}
$$

When time constant of the amplifier is very small compared to the amplitude $V(t)$ and phase $\theta(t)$, then for narrow band application i.e $<1.2 \mathrm{MHz}$, these amplitude and phase variations can be neglected and assuming it to be constant [7], But for high memory amplifiers it comes in the form of memory effects. Memory effects can be described as changes in the amplitude and phase of the output signal as function of the input signal amplitude and can be expressed as

$$
Z_{o}(t)=V_{0}\left(V _ { \text { in } ) } \cdot \operatorname { c o s } \left[\omega_{c} t+\theta+\phi_{\text {out }}\left(V_{\text {in })}\right]\right.\right.
$$

Whose complex envelop will be

$$
A_{\text {out }}(t)=V_{\text {out }}\left(V_{\text {in })} \cdot e^{j\left[\theta+\phi_{\text {out }}\left(V_{\text {in }}\right)\right]}\right.
$$

This AM/AM conversion is used to evaluate the $1-\mathrm{db}$ compression point.

\subsection{Nonlinearity of Power Amplifier in Two-Tone Analysis}

Let the two-tone input signal to the power amplifier is

$$
\begin{gathered}
Z_{i n}(t)=V_{i n} \cos _{\omega 1}(t)+V_{i n} \cos _{\omega 2}(t) \\
\omega_{1}<\omega_{2}, \omega_{2}-\omega_{1}=\Delta \omega
\end{gathered}
$$




$$
Z_{\text {in }}(t)=2 V_{\text {in }} \cos \left(\frac{\omega_{2}-\omega_{1}}{2} t\right) \cdot \cos \left(\frac{\omega_{2}+\omega_{1}}{2} t\right)
$$

Here frequency $\left(\frac{\omega_{2}-\omega_{1}}{2}\right)$ is the modulating frequency $\omega_{m}$, which is half of the frequency spacing between two tones. $\left(\frac{\omega_{2}+\omega_{1}}{2} t\right)$ is the RF center frequency. So equation (17) can be written as:

$$
Z_{i n}(t)=2 V_{i n} \cos \left(\omega_{m} t\right) \cdot \cos \left(\omega_{c e n} t\right)
$$

This two tone signal is recognized as double sideband suppressed carrier signal having carrier frequency $\omega_{c e n}$ and modulating frequency $\omega_{m}$. When this signal is applied to PA, the output signal can be expressed as:

$$
Z_{o}(t)=\sum_{k=0}^{K} a_{k} \cdot\left[V(t) \cdot \operatorname{Cos} \omega_{1} t+V(t) \cos \omega_{2} t\right]^{k}
$$

By expanding equation (19), new frequency components will appear, which shows the non-linearity of the device and called inter-modulation distortion [7]. In two-tone signal these are computed as:

$$
\omega_{\text {new }}=\left| \pm \alpha . \omega_{1} \pm \beta . \omega_{2}\right|
$$

Where $\alpha$ and $\beta$ are positive integers including zero. $\alpha+\beta=k$ denotes the order of the inter-modulation distortion.

To examine inter-modulation products, two frequencies $\omega_{1}$ and $\omega_{2}$, some of the orders of intermodulation products have been considered. To define the order, harmonic multiplying constants have been added with two frequencies producing the inter-modulation product. For example $\left(\omega_{1}+\omega_{2}\right)$ is second order, $\left(2 \omega_{1}-\omega_{2}\right)$ is third order, $\left(3 \omega_{1}-2 \omega_{2}\right)$ is fifth order, \& so on. For $\omega_{1}$ and $\omega_{2}$ to be two frequencies of $200 \mathrm{kHz}$ and $201 \mathrm{kHz}$ which are $1 \mathrm{kHz}$ apart, table 1 is showing the inter-modulation products.

Table 1-Intermodulation products

\begin{tabular}{|c|c|c|c|c|}
\hline \multirow{2}{*}{ Order } & Freq. 1 & Freq. 2 & $\begin{array}{c}\text { Freq. 1 } \\
\text { (kHz) }\end{array}$ & $\begin{array}{c}\text { Freq. 2 } \\
(\mathbf{k H z})\end{array}$ \\
\hline First & $\omega 1$ & $\omega 2$ & 200 & 201 \\
\hline Second & $\omega 1+\omega 2$ & $\omega 2-\omega 1$ & 401 & 1 \\
\hline \multirow{2}{*}{ Third } & $2 \omega 1-\omega 2$ & $2 \omega 2-\omega 1$ & 199 & 202 \\
\hline \multirow{2}{*}{ Fourth } & $2 \omega 1+\omega 2$ & $2 \omega 2+\omega 1$ & 601 & 602 \\
\cline { 2 - 5 } & $2 \omega 1+\omega 2$ & $2 \omega 2-2 \omega 1$ & 802 & 2 \\
\hline \multirow{2}{*}{ Fifth } & $3 \omega 1-\omega 2$ & $3 \omega 2-2 \omega 1$ & 198 & 203 \\
\cline { 2 - 5 } & $3 \omega 1+2 \omega 2$ & $3 \omega 2+2 \omega 1$ & 1002 & 1003 \\
\hline
\end{tabular}

From table 2, only the odd order inter-modulation products are close to the two fundamental frequencies $\omega_{1}$ and $\omega_{2}$. One third order product $\left(2 \omega_{1}-\omega_{2}\right)$ is $1 \mathrm{kHz}$ lower in frequency than $\omega_{1}$ and another third order product $\left(2 \omega_{2}-\omega_{1}\right)$ is $1 \mathrm{kHz}$ above $\omega_{2}$. One fifth order product $\left(3 \omega_{1}-2 \omega_{2}\right)$ is $2 \mathrm{kHz}$ below $\omega_{1}$ and another $\left(3 \omega_{2}-2 \omega_{1}\right)$ is $2 \mathrm{kHz}$ above $\omega_{2}$.

Table 2-Odd order products

\begin{tabular}{|c|c|c|c|c|}
\hline Order & Freq. 1 & Freq. 2 & $\begin{array}{c}\text { Freq. 1 } \\
(\mathbf{k H z})\end{array}$ & $\begin{array}{c}\text { Freq. 2 } \\
(\mathbf{k H z})\end{array}$ \\
\hline Third & $2 \omega 1-\omega 2$ & $2 \omega 2-\omega 1$ & 199 & 202 \\
\hline Fifth & $3 \omega 1-\omega 2$ & $3 \omega 2-2 \omega 1$ & 198 & 203 \\
\hline Seventh & $4 \omega 1-3 \omega 2$ & $4 \omega 2-3 \omega 1$ & 197 & 204 \\
\hline Ninth & $5 \omega 1-4 \omega 2$ & $5 \omega 2-4 \omega 1$ & 196 & 205 \\
\hline
\end{tabular}

In fact the odd order products are closest to the fundamental frequencies $\omega_{1}$ and $\omega_{2}$. The in-band IMD products cannot be easily filtered out [8]. The most commonly used measure of IMD is the ratio of the largest IMD (almost third order IMD) to the one of the two tone. For complex input signals nonlinearity appears over a continuous band of frequencies and is referred as spectral regrowth.

\section{INDIRECT LEARNING ARCHITECTURE}

The basic algorithm taken for this work is memory polynomial model. There are two methods to extract the power amplifier coefficients. One method is to model the power amplifier and then find its inverse. Since memory effects are also considered, so it is not possible to extract the exact inverse of the non-linear system. Second method is to use indirect learning architecture directly because it eliminates the assumptions about the model parameter estimation of the PA. The general form of a baseband memory polynomial PA model can be written as:

$$
Z_{\text {out }}(n)=\sum_{q=0}^{Q} \sum_{k=1}^{K} W_{k q} \cdot x_{n}(n-q)^{2 k-1}
$$

where $W_{k q}$ are complex memory polynomial coefficients. An integer value is given to $\mathrm{k}$ as $\mathrm{k}=0,1,2,3---\mathrm{K} . x_{n}$ and $Z_{\text {out }}(n)$ are the measured discrete input and output complex envelope signals of nth sample. $q=0,1,2,3-----Q$ is the memory interval and equal to sampling interval $\mathrm{T}$. $\mathrm{Q}$ is the maximum memory and $\mathrm{K}$ is the maximum polynomial order [9]. Since even terms are far away from the center frequency, only odd terms are considered.

Let

$$
U_{k q}(n-q)=x_{n}(n-q)^{2 k-1}
$$

By substituting equation (22) in equation (21), 


$$
Z_{\text {out }}(n)=\sum_{q=0}^{Q} \sum_{k=1}^{K} W_{k q} U_{k q}(n-q)
$$

Let

$$
U_{q}=\sum_{k=1}^{K} W_{k q} U_{k q}(n-q)
$$

By using equation (22)

$$
U_{q}=\sum_{k=1}^{K} W_{k q} x_{n}(n-q)^{2 k-1}
$$

After substituting this value in equation (23),

$$
Z_{\text {out }}(n)=\sum_{q=0}^{Q} U_{q}(n-q)
$$

Equation (21) can be represented as memory polynomial model with unity delay taps as shown in figure 2 . The unity delay taps are denoted by the symbol $Z^{-1}$. When $Z^{-1}$ delay tap is applied to the sequence of discrete digital values [1011]; the tap gives the previous value in the sequence and introduces a delay of one sampling interval q. Apply the value $Z^{-1}$ to the input value $x_{n}$ gives the previous input $x_{n}(n-1)$.

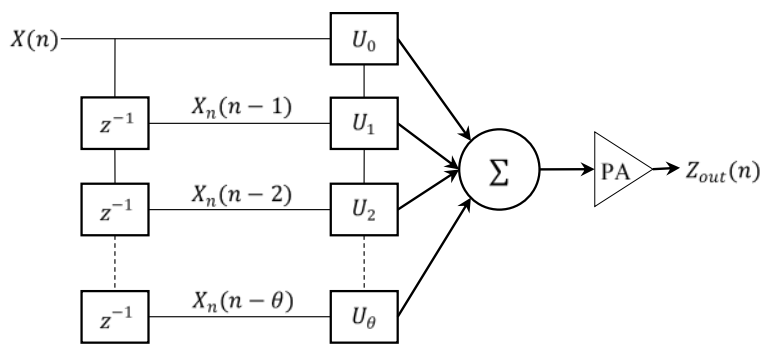

Fig 2: Memory Polynomial Model with Unity Delay

$$
Z^{-1} x_{n}=x(n-q)=x(n-1)
$$

Substitute value of $q$ in equation (23)

$$
\begin{aligned}
& Z_{\text {out }}(n)=\sum_{k=1}^{K} W_{k 0} U_{k 0}(n)+\sum_{k=1}^{K} W_{k 1} U_{k 1}(n-1)+\ldots . \\
& \sum_{k=1}^{K} W_{k Q} U_{k Q}(n-Q)
\end{aligned}
$$

$$
Z_{\text {out }}(n)=U_{0}+U_{1}+U_{2}+----U_{Q}
$$

By substituting values of $\mathrm{k} \& \mathrm{q}$ in equation (23),

$$
\begin{aligned}
& Z_{\text {out }}(n)=W_{10} U_{10}(n)+W_{20} U_{20}(n)+\ldots . . \\
& W_{k 0} U_{k 0}(n)+W_{11} U_{11}(n)+W_{21} U_{21}(n)+\ldots . . \\
& W_{k 1} U_{k 1}(n-1)+\ldots . . \\
& W_{1 Q} U_{1 Q}(n)+W_{2 Q} U_{2 Q}(n)+\ldots . \\
& W_{k Q} U_{k Q}(n-Q)
\end{aligned}
$$

$$
Z_{\text {out }}=U . W
$$

Where

$$
Z_{\text {out }}=\left[Z_{\text {out }}(0), Z_{\text {out }}(1), Z_{\text {out }}(2)-------Z_{\text {out }}(n-1)\right]^{T}
$$

$W=\left[\begin{array}{l}W_{10}, W_{20},------W_{k 0}, \\ W_{11}, W_{21},------W_{k 1}, \\ ------------ \\ W_{1 Q}, W_{2 Q},------W_{k Q},\end{array}\right]^{T}$

$U=\left[\begin{array}{l}U_{10}, U_{20},-----U_{k 0}, \\ U_{11}, U_{21},-----U_{k 1,}, \\ ----------- \\ U_{1 Q}, U_{2 Q},-----U_{k Q},\end{array}\right]$

\section{SIMULATION AND RESULTS}

In order to reduce the effect of non-linearity simulations are done by using MATLAB. Main channel power of actual amplifier is taken as 64.4691. ACPR of actual amplifier is taken as lower ACPR2 $=-47.0499$, lower ACPR $1=-59.4759$, Upper ACPR2 $=-46.5342$, Upper ACPR1 $=$-60.7407.Adjacent channel power of actual amplifier is taken as $17.4192 \mathrm{dbc}$, $4.9932 \mathrm{dbc}, 17.9349 \mathrm{dbc}$ and $3.7284 \mathrm{dbc}$. Training length is taken as 20,000. Polynomial order and memory length is varied for simulation. ACPR of modeled PA is measured and calculated error after simulation is shown in table 3 . Figure 3 shows the simulation results. The power spectral density of the input of the amplifier, output of the amplifier without predistortion and output of the amplifier with predistortion is shown in Figure 3

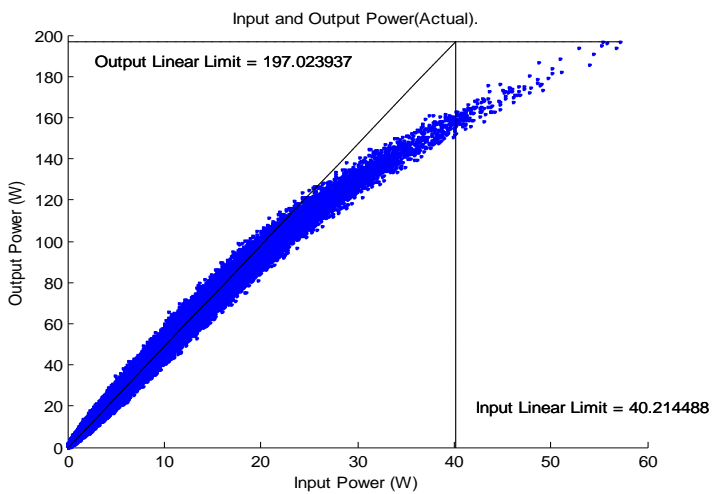

Fig 3: Power spectral density of PA with predistorter

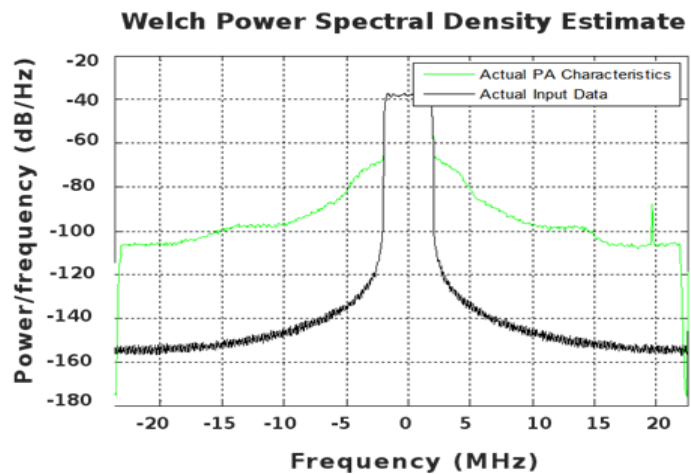

Fig 4: Adjacent channel power after linearization using memory polynomial PA

Equation (30) can be written in matrix form as 
As seen in the table 3, the best linearization is obtained when the memory depth is 2 and order is 7 , because for this combination error is minimum. Once it reaches its optimal polynomial order, the increase in order doesn't affect the linearization but increase in depth will increase the error rate of the amplifier. Due to this, spectrum of modeled PA will go away from the main PA, which increases the band width of the spectrum.

Table 3: ACPR measurements for different memory length and polynomial degree

\begin{tabular}{|c|c|c|c|c|c|c|c|}
\hline \multirow{2}{*}{$\begin{array}{c}\text { Memory } \\
\text { length }\end{array}$} & \multirow{2}{*}{$\begin{array}{c}\text { Polynomial } \\
\text { Degree }\end{array}$} & $\begin{array}{c}\text { PA Channel } \\
\text { Power } \\
\text { (Modeled) }\end{array}$ & \multicolumn{4}{|c|}{ Adjacent Channel Power Ratio (modeled) } \\
\cline { 5 - 8 } & & LOWER & $\begin{array}{c}\text { ACPR 1 } \\
\text { LOWER }\end{array}$ & $\begin{array}{c}\text { ACPR 1 } \\
\text { UPPER }\end{array}$ & $\begin{array}{c}\text { ACPR 2 } \\
\text { UPPER }\end{array}$ & Error \\
\hline 2 & 6 & 64.4921 & -12.4334 & 16.5687 & 17.2351 & -12.3110 & .3732 \\
\hline 2 & 7 & 64.4921 & -12.319 & 16.5755 & 17.2502 & -12.9913 & -3716 \\
\hline 3 & 6 & 64.4918 & -10.6589 & 17.4099 & 17.9965 & -12.2707 & .3741 \\
\hline 3 & 5 & 64.4919 & -9.0804 & 17.3240 & 17.8824 & -9.4474 & .3763 \\
\hline 4 & 7 & 64.4918 & -11.3284 & 17.4706 & 18.0493 & -11.8459 & .3727 \\
\hline
\end{tabular}

\section{CONCLUSION}

This paper proposes a memory polynomial modelling of Power amplifier, whose novelty consists in enhancing modelling accuracy by means of different combinations of nonlinear order and memory depth. Simulation results have been analyzed by taking the considerations of the effects of different amount of memory depth and nonlinear order. It has been observed that the modelling accuracy can be improved by suitable combination of memory and nonlinear order. The result shows that error in modeling is minimum (0.3716) when memory depth is 2 and nonlinear order is 7 .

\section{REFERENCES}

[1] Steve C. Cripps, Advanced Techniques in RF Power Amplifier Design Chapter 3.

[2] Peter B. Kenington, Linearity RF Amplifier Design, chapter 2.

[3] C. Lee, V. Postoyalko, and T. O'Farrell, "Analysis and Simulation of AM-AM/PM Nonlinear Distortion due to Direct Laser Modulation in Radio over Fiber Systems", International Topical Meeting on Microwave Photonics, pp. 137-140, Nov. 2002.

[4] Sang-Woong Yoon, "Static and Dynamic Error Vector Magnitude Behavior of 2.4-GHz Power Amplifier", IEEE Tran. Microwave Theory Tech vol. 55, no. 4, p.647 (2007).

[5] R. Sperlich, J.A. Sills, and J.S. Kenney, "Power amplifier linearization with memory effects using digital pre- distortion and genetic algorithms", IEEE Radio and Wireless Conference, RAWCON 2004, September 2004.

[6] D. Morgan, Z. Ma, J. Kim, M. Zierdt, and J. Pastalan, “A Generalized Memory Polynomial Model for Digital Predistortion of RF Power Amplifiers", IEEE Transactions on Signal Processing, vol. 54, no. 10, pp. 3852-3860, Oct. 2006.

[7] N. Borges De Carvalho and J. C. Pedro, "Two-tone IMD asymmetry in microwave power amplifiers," IEEE MTTS Int. Microwave Symp. Dig., 2000, pp. 445-448.

[8] H. Ku, M. D. Mckinley and J. S. Kenney, "Extraction of accurate behavioral models for power amplifiers with memory effects using two-tone measurements," IEEE MTT-S Int. Microwave Symp. Dig., 2002, pp.139-142.

[9] MORGAN, D. R.MA, Z. KIM, J.ZIERDT and M. G. PASTALAN, "A Generalized Memory Polynomial Model for Digital Predistortion of RF Power Amplifiers", IEEE Trans. Signal Processing, 54 No. 10 (Oct 2006), 3852-3860.

[10] L. Ding, G. Zhou and D. Morgan, "A Robust Digital Baseband Predistorter Constructed Using Memory Polynomials", IEEE Trans. Comm., vol. 52, no. 1, pp. 159-165, Jan. 2004.

[11] Moon, J., and Kim, B.: "Enhanced Hammerstein behavioral model for broadband wireless transmitters", IEEE Trans. Microwave Theory Tech., (4), pp. 924-933, Apr. 2011 